\section{Cellular environment and freedom of gene expression}

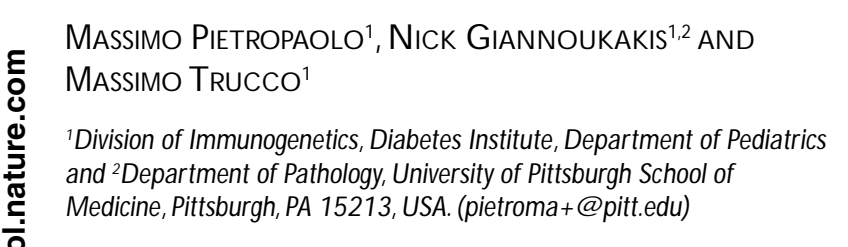

Data reported by Derbinski et al. ${ }^{1}$ reinforce the concept that thymic cells express a variety of tissue-restricted antigens and may be actively involved in negative selection. Based mainly on results obtained by flow cytometric analysis of cells derived from primary thymic tissue, the authors claim that the expression of a number of tissue-restricted proteins in medullary thymic epithelial cells (mTECS) appeared heterogeneous and that high expression of these molecules was restricted to a minor fraction of mTECS. One conclusion is that mTECs seem sufficient, but not necessary, to induce CD4 $\mathrm{T}$ cell tolerance ${ }^{1}$.

In our view-and despite their conclusions-Derbinski et al. did not entirely rule out the possibility that other thymic cells, in addition to mTECS, can express a diverse range of tissue-specific antigens. Indeed, published observations show that some tissue-restricted antigens associated with autoimmune diabetes, including insulin and glutamic acid decarboxylase (GAD), are also expressed in the thymus by DCs and macrophages ${ }^{2,3}$. In addition, studies in mice have shown that the thymus contains cells, termed peripheral antigen-expressing (PAE) cells, that express insulin, and experiments in transgenic mice provide evidence that these PAE cells may have a tolerogenic function $^{3-6}$. Similar cells expressing islet cell antigens such as insulin (proinsulin), GAD and the neuroendocrine antigen IA-2 have been described both in human thymic and peripheral lymphoid tissues ${ }^{3}$.

We favor the concept that microenvironmental effects - such as dynamic cytokine, growth factor and cell-cell contact fluxes in the thymus - are essential in modulating gene expression. Consequently, the complex cytokine and cell adhesion networks in the thymic microenvironment can modify the expression of tissue-restricted antigen expression. Similar effects have been shown in a number of other microenvironments ${ }^{7-9}$. In the study by Derbinsky et al. it is not clear whether DCs and other non-mTECs were cultured before sorting or whether tissue-restricted antigen expression by all cell populations under a variety of cytokine conditions that simulated different thymic microenvironments were evaluated ${ }^{1}$. We have compelling evidence that expression of tissue-restricted antigenssuch as insulin and the ICA69 (islet cell autoantigen $69 \mathrm{kD}^{10}$ ) in murine DCs - as well as insulin promoter activity in murine DCs varies dramatically depending on the cytokine mixture used in culture ${ }^{11}$; it can range from high expression to a complete absence $(\mathrm{N}$. Giannoukakis et al. and M. Pietropaolo et al., unpublished data).

The thymic medulla provides a framework from which thymocytes can mature. Based on our findings, it would be reasonable to expect that not only mTECs, but also DCs, may play some role in either further nurturing or directing $\mathrm{T}$ cells to the deletion pathway within the thymic medullar compartment ${ }^{12}$. Derbinski et al. have provided some insights into this process, but more work is needed to determine whether different players within the thymic microenvironment influence the freedom of expression of their constituents.

\footnotetext{
1. Derbinski, J., Schulte,A., Kyewski, B. \& Klein, L. Nature Immunol. 2, 1032-1039 (2001).

2. Throsby, M. et al. Endocrinology 139, 2399-2406 (1998).

. Pugliese, A. et al. J. Clin. Invest. 107, 555-564 (2001).

Smith, K. M., O Ison, D. C., Hirose, R. \& Hanahan, D. Int. Immunol. 9, 1355-1365 (1997).

5. Ardavin, C. Immunol.Today 18, 350-361 (1997).

6. Faratian, D., Colvin, B. L., O 'Connell, P. J., Morelli, A. E. \& Thomson, A.W. Graft 3, 54-58 (2000).

7. Roskelley, C. D., Srebrow,A \& Bissell, M. J. Curr. Opin. Cell Biol. 7, 736-747 (1995).

8. Radinsky, R. Cancer M etastasis Rev. 14, 323-338 (1995).

9. Savino,W., de Mello-Coelho,V. \& Dardeene, M Neuroimmunomodulation 2, 313-318 (1995).

10. Pietropaolo, M. et al. J. Clin. Invest. 92, 359-371 (1993).

11. Inaba, K. et al. J. Exp. Med. 176, 1693-1702 (1992).

12. Lo, D., Reilly, C. R., D eKoning, J., Laufer, T. M. \& G limcher, L. M. Immunol. Rev. 16, 3-14 (1997).
}

\section{Lymphoid organs contain diverse cells expressing self-molecules}

\section{Alberto Pugliese and Juan Diez}

Immunogenetics Program, Diabetes Research Institute, University of M iami School of M edicine, M iami, FL 33136, USA. (apuglies@ med.miami.edu)

Derbinski et al. ${ }^{1}$ reported the expression of self-molecules in the mouse thymus. Their work follows earlier reports showing that self-molecules with tissue-restricted expression (or "peripheral" proteins) are also produced in the thymus through "ectopic" or "promiscuous" transcription and translation ${ }^{2-5}$ and that such expression contributes to shaping a self-tolerant $\mathrm{T}$ cell repertoire $^{6}$. Derbinski et al. used RT-PCR to detect peripheral protein transcripts in thymic cell populations purified by fluorescence-activated cell sorting after staining with markers specific for medullar or cortical thymic epithelial cells (mTECs and cTECs, respectively) and for bone marrow-derived APCs such as macrophages and DCs. Essentially all the molecules studied-including several autoantigens involved in autoimmune diseases such as type I diabetes and multiple sclerosis - were expressed by mTECs and not by DCs or macrophages.
These findings contrast with earlier reports $^{3,5}$, which showed that thymic cells expressing type I diabetes autoantigens (proinsulin and insulin, GAD and IA-2 and other pancreatic hormones) had surface markers that are typical of DCs and macrophages in human and mouse thymi, respectively. In our experiments, immunohistochemistry and double-immunofluorescence techniques were used to stain frozen tissue sections ${ }^{5}$. This approach prevents the detection of artifacts potentially associated with tissue processing and cell manipulation and allows the examination of cells in their native state. Unlike Derbinski et al. ${ }^{1}$, but like Throsby et al. ${ }^{3}$, we were unable to colocalize proinsulin, GAD and IA-2 with cytokeratin (a marker of thymic epithelial cells) ${ }^{5}$. We were also unable to colocalize proinsulin with $\mathrm{AIRE}^{5}$, which is expressed in thymic epithelial cells and a subset of DCs? ${ }^{7}$. If our findings were based on 